\title{
Multicolour observations, inhomogeneity and evolution
}

\author{
Charles Hellaby* \\ Department of Mathematics and Applied Mathematics, University of Cape Town, Rondebosch, 7701, South Africa
}

Received 31 October 2000 / Accepted 30 January 2001

\begin{abstract}
We propose a method of testing source evolution theories that is independent of the effects of inhomogeneity, and thus complementary to other studies of evolution. It is suitable for large scale sky surveys, and the new generation of large telescopes. In an earlier paper it was shown that basic cosmological observations luminosity versus redshift, area distance versus redshift and number counts versus redshift - cannot separate the effects of cosmic inhomogeneity, cosmic evolution and source evolution. We here investigate multicolour observations, and show that by comparing luminosity versus redshift in two or more colours, contraints can be placed on source evolution even if unknown source evolution is present, providing an important test of evolution theories that is complementary to present methods. However, number counts in different colours versus redshift are not useful in separating the effects of source evolution and inhomogeneity.
\end{abstract}

Key words. large scale structure of Universe - galaxies: evolution

\section{Introduction}

As measurements of the Cosmos become more extensive and more accurate, it becomes increasingly important to take account of the evolution of galaxies and clusters of galaxies, since these have a direct effect on our cosmological measurements. It seems that the Hubble constant may soon be well known, and limits on the acceleration parameter may improve rapidly. The next step is to estimate deviations from homogeneity on the large scale ${ }^{1}$. It is expected that the Sloan digital sky survey (SDSS) will detail the galaxy distribution out to $z \leq 0.5$. How does this data relate to the spacetime metric?

It was previously shown by Mustapha et al. (1997) (MHE) that observations of luminosity versus redshift and area distance (or luminosity distance) versus redshift cannot distinguish between the effects of source evolution, cosmic inhomogeneity, and cosmic evolution. Riess et al. (1998) and Perlmutter et al. (1999) have fitted supernova observations to an FLRW model with non-zero $\Lambda$, but Celerier (2000) has shown they could equally well be fitted by an inhomogeneous model with zero $\Lambda^{2}$.

Whilst the Copernican Principle may lead many to assert that homogeneity is valid on a large enough scale, it

\footnotetext{
* e-mail: cwh@maths.uct.ac

1 For example, Wang et al. (1998) have estimated that variations of up to $10 \%$ between local measures of $H_{0}$ and its large scale value are possible.

2 Also Maor et al. (2000) argue these observations cannot determine the future fate of the universe.
}

is better not to assume something if it can be measured. In addition, the observed luminosity versus redshift relation does not fit the FLRW predictions without some adjustments, such as a cosmological constant that has a problematic value, or source evolution. And since source evolution is really not known, one should be suspicious of the results. Thus it has never been observationally determined on which scale homogeneity is valid ${ }^{3}$.

Galaxy evolution is now a very active field, but there is a long way to go $^{4}$. There are many functions to be determined - the rate of formation of each galaxy type, the rate of formation of stars of different masses in each galaxy type the rate of galaxy mergers ${ }^{5}$, the effect of galaxy mergers and encounters on star formation, how central bulges and bars form, how low surface brightness galaxies fit into the picture, etc. The early appearance of galaxies and the relationship between quasars and galaxies is really not known.

${ }^{3}$ For example, Barrett \& Clarkson (2000) have shown that Stephani models with significant inhomogeneity can fit a range of key cosmological observations.

${ }^{4}$ See e.g. Ellis et al. (2000) and references therein, Kennicut (1998), Ellis (1997), Impey \& Bothun (1997), Bell \& Bower (2000), Blundell \& Rawlings (1999), Brunner et al. (2000), Bullock et al. (2001), Bunker et al. (2000), Cavaliere \& Vittorini (2000), Dickinson (2000), Fasano et al. (2000), Genzel et al. (1998), Kodama \& Bower (2001), Ponman et al. (1999), Watts \& Taylor (2000).

${ }^{5}$ It has also been proposed that stellar mergers may be significant in dense clusters. 
Many studies of source evolution make an assumption of homogeneity at some point, by using observational relations derived in a FLRW model. While we don't dispute their usefulness, the danger is that the resulting source evolution relations may later be used to demonstrate homogeneity. Thus methods of determining source evolution that don't make assumptions about the universe model should be emphasised as more reliable. This point was also made by Goodman (1995). Observations of supernovae (Riess et al. 1998; Perlmutter et al. 1999) may help to separate inhomogeneity from source evolution, out to distances where their light curves can be measured, though it is still uncertain whether they are free of evolution effects (e.g. Riess et al. 1999).

As a contribution towards this requirement, we investigate whether multicolour observations can help to separate inhomogeneity from source evolution. The measurements in different colours may be a selection of spectral line intensities or luminosties in $U, B$ and $V$ filters, say. In any case, one needs to know the absolute luminosity in each colour at each $z$ value, so there is a new source evolution function for each colour. Thus it seems that multicolour observations may not improve the situation. However, a key point is that the luminosity distance must be the same in all wavelengths for each given source. So if the luminosity-redshift plot in two colours is not the same, this is evidence that the two colours have different evolution functions. This approach is used below, and it is found that the basic uncertainty in absolute values remains, but the luminosity-redshift relations in different colours must be related to each other, thus providing constraints on evolution theories.

The choice of cosmological model is not central to the ideas presented here, but to keep the equations simple and focus on the basic concepts, we choose the simplest inhomogeneous cosmological solution of Einstein's equations. This is the Lemaitre-Tolman (LT) model ${ }^{6}-$ a spherically symmetric dust cosmology that is inhomogeneous in the radial direction. If one accepts that the large scale universe is a collection of galaxies with negligible bulk rotation and interacting only through gravity, then the dust equation of state is valid, and the cosmic time evolution is pretty much determined along each worldline. For reasons of simplicity we assume the observer is at the centre; thus we are assuming large scale isotropy about the milky way or some nearby point. Isotropy is relatively easy to test and is not a bad assumption on large angular scales. It is also a natural simplification to make, since we are at the centre of the null cone we observe. A more general approach would cloud the issue at hand, though it will be needed in the long run. More importantly, the degree of deviation from isotropy is directly seen and is not mixed up with source evolution or cosmic evolution.

\footnotetext{
6 Though it is sometimes called the Lemaître-Tolman-Bondi (LTB) model, Krasinski's (1997) terminology is adopted here.
}

\section{The cosmological model}

We summarise the results of MHE, as background to the present considerations, and refer the reader to the papers cited there.

The Lemaître-Tolman (LT) metric (Lemaître 1933; Tolman 1934; Bondi 1947) is

$\mathrm{d} s^{2}=-\mathrm{d} t^{2}+\frac{\left(R^{\prime}\right)^{2}}{1+2 E} \mathrm{~d} r^{2}+R^{2} \mathrm{~d} \Omega^{2}$

where $R=R(t, r), E=E(r), R^{\prime}=\partial R / \partial r$, and $\mathrm{d} \Omega^{2}=$ $\mathrm{d} \theta^{2}+\sin ^{2} \theta \mathrm{d} \phi^{2}$. The function $R$ is the areal radius, which obeys

$\dot{R}^{2}=\frac{2 M}{R}+2 E$

and the arbitrary function $E$ determines both the local geometry and the local type of time evolution. The arbitrary function $M=M(r)$ plays the role of the total gravitational mass interior to shells of coordinate radius $r$. This has solutions in terms of the parameter $\eta$

$R=\frac{M}{\mathcal{E}} \phi_{0}(\eta), \quad \xi(\eta)=\frac{(\mathcal{E})^{3 / 2}\left(t-t_{B}\right)}{M}$

where

$$
\begin{aligned}
& \mathcal{E}(r)=\left\{\begin{array}{l}
2 E(r), \\
1, \\
-2 E(r),
\end{array} \quad \phi_{0}(\eta)=\left\{\begin{array}{l}
\cosh \eta-1, \\
\eta^{2} / 2, \\
1-\cos \eta,
\end{array}\right.\right. \\
& \xi(\eta)=\left\{\begin{array} { l } 
{ \operatorname { s i n h } \eta - \eta , } \\
{ \eta ^ { 3 } / 6 , } \\
{ \eta - \operatorname { s i n } \eta , }
\end{array} \quad \text { when } \quad \frac { R E } { M } \left\{\begin{array}{l}
>0, \\
=0, \\
<0,
\end{array}\right.\right.
\end{aligned}
$$

and $t_{B}=t_{B}(r)$ is a third arbitrary function. It gives the time of the big bang, $R=0$, locally. Although the initial singularity is spacelike everywhere ${ }^{7}$ the spacetime emerges from the initial singularity over a finite or possibly infinite time. The density is

$8 \pi \rho=\frac{2 M^{\prime}}{R^{2} R^{\prime}}$

Putting the observer at the centre of symmetry, $r=0$, the observer's past null cone is the solution of

$\mathrm{d} t=\frac{-R^{\prime}}{\sqrt{1+2 E}} \mathrm{~d} r$

that passes through $r=0$ at $t=t_{0}$ - i.e. at the present. MHE wrote this particular solution as $\hat{t}=\hat{t}(r)$, and a hat will henceforth indicate quantities evaluated on this null cone. Since we really only need this one path, MHE used the freedom in the radial coordinate $r$ to specify

$R^{\prime}=1$

on $t=\hat{t}(r)$ only. This greatly simplifies the equations to be solved. The path of the light cone is then

$\hat{t}(r)=t_{0}-r$.

7 Except where there's a shell-focussing singularity. 
MHE then went on to show how the 3 arbitrary functions of the LT model, $E(r), M(r)$ and $t_{B}(r)$, could be determined from observations, if one knew the source evolution. The observations needed were the source number counts against redshift $z$, and the diameter distance $R$ (or the related luminosity distance) against $z$. The evolution functions needed were the mass per source, and the absolute diameter or luminosity, both against $z$. Given only these observations, it was shown that both source evolution and inhomogeneity cause deviations from the expected FLRW observational relations ${ }^{8}$, and there is no way to distinguish the two.

We now generalise the MHE approach to observations in two or more different colours.

\section{Observing model}

\subsection{Assumptions}

We assume the following:

- The universe is isotropic about the earth to good approximation on the large scale;

- Small scale inhomogeneity has been eliminated by averaging cosmic observables over the whole sky, so that they are functions of $z$ only;

- There are $J$ types of visible sources, $1 \leq j \leq J$, e.g. spirals, ellipticals, Abell clusters, field galaxies, etc. The types have different intrinsic properties and may evolve differently - i.e. have different source evolution functions. Mergers could correspond to the removal of certain types in favour of new types. We assume the various types can be reliably distinguished - but see the discussion in the last point of Sect. 3.3 and in Sect. 6.3;

- There are $I$ different spectral frequencies or colour filters being used for observing, $1 \leq i \leq I$, e.g. $U, B$ and $V$ filters, or preferably a set of spectral line intensities;

- All wavelengths experience the same delay and the same cosmological redshift due to the geometry and evolution of the universe ${ }^{9}$.

\subsection{Notation}

Whether the colour measurements are spectral line intensities or colour filter apparent magnitudes, we will call them "colours".

\subsubsection{Observables}

The following "observables" are smooth functions fitted to data from direct observations, that have been corrected for selection effects, absorbtion and other effects mentioned below.

\footnotetext{
${ }^{8}$ Examples of strong deviations from the standard FLRW observational relations in models with realistic amounts of inhomogeneity were given in Mustapha et al. (1998) (MBHE).

9 However redshifts of individual objects due to peculiar motions will differ, as noted below.
}

$-z=$ measured redshift of each object;

$-n_{j}(z)=$ observed number density of source type $j$ in redshift space, per steradian per unit redshift interval, $1 \leq j \leq J$

$-\ell_{j i}(z)=$ measured apparent luminosity of source type $j$ in colour $i$ at redshift $z, 1 \leq i \leq I$;

$-\delta_{j i}(z)=$ measured angular diameter of source type $j$ in colour $i$ at redshift $z$.

\subsubsection{Theoretical quantities}

The following are functions that must be supplied by a theory of source evolution.

- $L_{j}(z)=$ absolute bolmetric luminosity of source type $j$, at the epoch corresponding to redshift $z$;

- $L_{j i}(z)=$ absolute luminosity of source type $j$, in colour $i$, at $z$;

- $D_{j i}(z)=$ proper diameter of source type $j$, in colour $i$, at $z$;

- $m_{j}(z)=$ total mass associated with (gravitationally bound to) source type $j$;

$-\nu(z)=$ total proper density in redshift space of matter not associated with a luminous source.

\subsection{Complications}

There are of course many sources of error in making and reducing the observations, which need careful attention. However we will not go into them here, except to note the most important ones. It is assumed the observational functions have already been corrected for these effects. It is evident that measurements of spectral line intensities have fewer problems than luminosities through colour filters. If a program of observations were being planned, line intensities are a natural extension to redshift measurements, and would be the natural choice. However measurements of colour magnitudes are considerably easier to make, and more such data already exists.

- The redshift we are interested in is the cosmological one, but the actual redshift of a source is the combination of cosmological and peculiar velocity contributions, causing considerable scatter in the observed $z$ values from a given distance. Since the functions of $z$ we use here are all-sky averages, this is not a problem. However, in generalising to models with a measure of anisotropy in addition to radial inhomogeneity, a net "peculiar velocity" on the averaging scale being considered, should rather be viewed as an inhomogeneity in the cosmological expansion rate ${ }^{10}$;

${ }^{10}$ However, as was shown in MBHE, inhomogeneities near the maximum in $\hat{R}(z)$ can create loops in the $\hat{R}(z)$ graph, so that objects at different distances have the same $z$. This is very like the "finger of god" effect. Thus, if this were common, sources selected by $z$ may not all be at the same true distance, and there would be a blurring of source properties near the maximum, in addition to any observational uncertainties. 
- The observed colours will be redshifted. If spectral line intensities are used, then the emitted frequencies will be known, but if colour filters are used, different parts of the true spectrum are selected at each $z$ value, so correcting for this could be quite tricky, to say the least ${ }^{11}$;

- Sources will suffer absorbtion and reddening. This could be problematic as intergalactic absorbtion is not all that well known, even at low $z$ values. Ideally wavelengths should be chosen to minimise absorbtion. Clearly, light from sources at high $z$ will experience a wider range of absorbtion effects on route to us;

- Selection effects are crucial to real observations (Ellis et al. 1984; Teerikorpi 1997; Totani \& Yoshii 2000). Only a fraction $f$ of sources are actually detected, and $f$ depends on a source's apparent size and surface brightness. Thus the observed number densities $n_{j}$ are related to the true number $\tilde{n}_{j}$ densities by

$n_{j}=f\left(\ell_{j i}, \delta_{j i}, z\right) \tilde{n}_{j}$

For quantities calculated from the $n_{j}$, this is a serious problem. But for those that depend on comparisons of measured $\ell_{j i}$ values - exactly what we need below there is no real problem;

- Source evolution is naturally a function of time at the source, $\tau$, but here it is written as a function of $z$. Although the age of the universe at each $z$ is an output function of the MHE algorithm, one needs the source evolution functions as initial inputs. An iterative correction process could be developed, once our knowledge of source evolution is adequate to the task;

- It would be difficult to identify the same source type at different stages of evolution, especially at high $z$ values, and especially if galaxy mergers are very frequent. For this reason it may well be best to initially consider a single source type - i.e. treat all sources as the same type - and test for the bulk evolution properties of the cosmic luminous matter content.

\section{Comparison of multicolour observations}

Multicolour observations cannot be directly used as input to the LT model (or any other metric), which only requires $\hat{R}(z)$ and $\hat{\rho}(z)$ (plus the coordinate condition $\widehat{R^{\prime}}=1$ ) to fully determine the metric. However for any given source, the ratio of apparent to absolute luminosity must be the same in all wavelengths. In other words, the luminosity distances obtained in each of the colours or spectral lines must be the same.

\footnotetext{
11 However, a well developed source evolution theory could make predicions for the relevant part of the spectrum according to $z$.
}

\subsection{Distances}

The luminosity distance is

$d_{\mathrm{L}}=\sqrt{\frac{L}{4 \pi \ell}}$.

Ideally, all estimates at any given $z$, for all source types and all colours, should agree, if we knew the $L_{j i}(z)$ completely:

$\frac{L_{j i}(z)}{4 \pi \ell_{j i}(z)}=d_{\mathrm{L}}^{2}(z) \quad \forall i, j$.

This is a set of $I \times J$ equations for the $I \times J+1$ unknown functions $L_{j i}(z)$ and $d_{\mathrm{L}}(z)$. Thus with sufficient observations we could determine the $L_{j i}(z)$ relative to each other, but none of them absolutely.

To get the function $\hat{R}(z)$ we take the average ${ }^{12}$

$\hat{R}(z)(1+z)=\bar{d}_{\mathrm{L}}, \quad \bar{d}_{\mathrm{L}}=\frac{\sum_{j=1}^{J}\left(\frac{1}{I} \sum_{i=1}^{I} \sqrt{\frac{L_{j i}}{4 \pi \ell_{j i}}} n_{j}\right)}{\sum_{j=1}^{J} n_{j}}$

In reality, observations would be of different reliability, and a weighted average would be used.

We have similar relations for the diameter distance

$d_{\mathrm{D}}=\frac{D}{\delta}$

all of which should agree, if we knew the $D_{j i}(z)$

$\frac{D_{j i}(z)}{\delta_{j i}(z)}=d_{\mathrm{D}}(z) \quad \forall i, j$.

Again the average should give a good estimate of the LT areal radius

$\hat{R}(z)=\bar{d}_{\mathrm{D}}=\frac{\sum_{j=1}^{J}\left(\frac{1}{I} \sum_{i=1}^{I} \frac{D_{j i}}{\delta_{j i}} n_{j}\right)}{\sum_{j=1}^{J} n_{j}}$

and no absolute diameters can be determined.

If both types of distance measurements are available, then we have a combined requirement:

$\frac{1}{(1+z)^{2}} \frac{L_{j i}(z)}{4 \pi \ell_{j i}(z)}=\hat{R}^{2}(z)=\left(\frac{D_{j i}(z)}{\delta_{j i}(z)}\right)^{2} \quad \forall i, j$

leaving only one free function out of the set $\left\{L_{j i}, D_{j i}\right\}$.

From here on we will focus on the luminosity distance, as the one that can be used to the greatest depth ${ }^{13}$.

\subsection{Densities}

If the number of sources of type $j$ observed between $z \&$ $z+\mathrm{d} z$ in solid angle $\mathrm{d} \Omega$ is

$n_{j} \mathrm{~d} \Omega \mathrm{d} z$

\footnotetext{
12 Equation (31) of MHE is incorrect.

13 A source's luminosity may be determined, even if it's size can't be resolved.
} 
then the net mass is

$n_{j} m_{j} \mathrm{~d} \Omega \mathrm{d} z$

so the combined mass of all source types seen over the whole sky is

$4 \pi \sum_{j=1}^{J} n_{j} m_{j} \mathrm{~d} z$

and if we allow for a distribution of non-visible matter that isn't associated with any source, we get the total mass as

$4 \pi\left(\sum_{j=1}^{J} n_{j} m_{j}+\nu\right) \mathrm{d} z$.

Now the local proper density in the LT model is

$\rho=\rho(t, r)$

and its value on the null cone is

$\hat{\rho}=\rho(\hat{t}(r), r)$.

The total mass between $r$ and $r+\mathrm{d} r$ is

$\hat{\rho} \widehat{d^{3} V}=\hat{\rho} \frac{4 \pi \hat{R}^{2} \widehat{R^{\prime}}}{\sqrt{1+2 E}} \mathrm{~d} r$

and hence

$\hat{\rho} \frac{\hat{R}^{2} \widehat{R^{\prime}}}{\sqrt{1+2 E}}=\left(\sum_{j=1}^{J} n_{j} m_{j}+\nu\right) \frac{\mathrm{d} z}{\mathrm{~d} r}$.

\subsection{Fitting the data with an $L T$ model}

Equations (24) and (12) or (15) or (16) show how the LT functions relate to the observables and the source evolution functions. Once the last two are known, the MHE procedure shows that, if we are given

$\left(\sum_{j=1}^{J} n_{j}(z) m_{j}(z)+\nu(z)\right) \quad$ and $\quad \bar{d}(z)$

then the 3 arbitrary functions that characterise the best fit LT model can be determined. Thus if we knew the source evolution, we could determine the LT model that reproduces the observations. In particular, we could determine how close the universe is to large scale homogeneity on the smoothing scale used.

\section{Number counts}

The number counts only provide a single function of $z$ for each source type, and so do not help us to distinguish inhomogeneity from source evolution. The advantage of the luminosity measurements is that each source is measured more than once in different frequencies.

\subsection{Colour-weighted number counts}

Number counts of field galaxies with different colours have been used to try and demonstrate source evolution. If such a method is undertaken, it is because the $\ell_{j i}$ are not known, and also because spectral line data is not available, only colour filter luminosities. We here try to model this process in an admittedly rough and ready manner - the number counts obtained in different colours are modelled by weighting the total number count by a function of all the colour luminosities of that source type.

Let

$-w_{j i}=$ weighting functions for each source type. These may in general depend on all $I$ of the colour luminosities, e.g. $w_{23}=w_{23}\left(L_{21}, L_{22}, L_{23}\right)$;

- $n_{j i}=$ number of source type $j$, counted in colour band $i$. Thus at each $z$ value:

$n_{j i}=w_{j i}\left(L_{j i}\right) n_{j}$;

$-c_{i}=$ colour-weighted number count of all source types in colour band $i$-i.e. the number density in redshift space $c_{i}(z)$ of all source types with colour $i$ is

$$
c_{i}=\sum_{j=1}^{J} n_{j i}=\sum_{j=1}^{J} w_{j i}\left(L_{j i}\right) n_{j} .
$$

The simplest weighting function is a step function with a cut-off luminosity below which the source is not detected,

$w_{j i}=\left\{\begin{array}{ll}1 & L_{j i}>L_{j i}^{0} \\ 0 & L_{j i} \leq L_{j i}^{0}\end{array}\right.$.

This means that each of the $c_{i}$ equals the total number count out to some $z$ value, and after that each source type disappears from the count fairly rapidly around some $z$ value. The $z$ values for each type and each colour are probably not very different. Again any variation could equally well be due to inhomogeneity in the population of source types as to source evolution. Add to this the difficulty of correcting for the redshifting of frequencies detected in each colour band, and it is clear that this data does not assist in distinguishing source evolution from inhomogeneity. Indeed, even if there were no large scale inhomogeneity, it is still difficult to get clear information about source evolution from this data ${ }^{14}$.

Now suppose the weighting function were proportional to the relative luminosity of that source type in that colour

$w_{j i}=\frac{\ell_{j i}}{\bar{\ell}_{j}}, \quad \bar{\ell}_{j}=\frac{1}{I} \sum_{i=1}^{I} \ell_{j i}$.

This is of course not realistic, but by presenting an excessively favourable case we highlight how unpromising the

14 For example, morphological studies suggest that the excess of faint blue galaxies is due to those with peculiar morphology. But which of the more modern nearby galaxy types they evolve into, if any, or whether this is actually a manisfestation of cosmic inhomogeneity, is not known. 
more realistic scenarios are. For these weighting functions we would have

$n_{j i}=\frac{\ell_{j i}}{\bar{\ell}_{j}} n_{j}=\frac{L_{j i}}{\bar{L}_{j}} n_{j}, \quad \bar{L}_{j}=\frac{1}{I} \sum_{i=1}^{I} L_{j i}$

and so the number density in redshift space $c_{i}(z)$ of all source types with colour $i$ would be

$c_{i}=\sum_{j=1}^{J} n_{j i}=\sum_{j=1}^{J}\left(\frac{L_{j i}}{\bar{L}_{j}} n_{j}\right)$

where $\bar{\ell}_{j}$ and $\bar{L}_{j}$ are the mean luminosities. Assuming the $n_{j}$ are known, we have $I$ linear relations involving $I \times J$ unknown coefficients. This certainly doesn't help us solve for the evolution functions, though it does place mild constraints on them. The ideal situation is if there is only one source type, in which case we have $I$ equations for $I$ unknowns.

Any realistic weighting function would smear together these equations, making it virtually impossible to solve for the the coefficients with any certainty.

These results, even for the most ideal case, are conditional on the number counts in each colour being tabulated against redshift $z$. But since they are actually summed over a large range of $z$, then the constraints are lost, as this effectively smooths out the evolution functions over time.

\section{Examples}

\subsection{1 type of source and 3 colours}

This is the case where we treat all galaxies as more or less the same, with roughly the same luminosity and colour evolution.

We have $J=j=1, I=3, i=1,2,3$, so all subscripts are colour subscripts. The constraints are

$\frac{L_{1}}{4 \pi \ell_{1}}=\frac{L_{2}}{4 \pi \ell_{2}}=\frac{L_{3}}{4 \pi \ell_{3}}=\bar{d}_{\mathrm{L}}^{2}(z)$

where all the $L \mathrm{~s}$ and $\ell$ s are functions of $z$, and

$\bar{d}_{\mathrm{L}}=\frac{1}{3}\left(\sqrt{\frac{L_{1}}{4 \pi \ell_{1}}}+\sqrt{\frac{L_{2}}{4 \pi \ell_{2}}}+\sqrt{\frac{L_{3}}{4 \pi \ell_{3}}}\right)$.

This gives effectively 2 constraints on 3 evolution functions - e.g. $L_{1} / L_{2}$ and $L_{2} / L_{3}$.

\subsection{2 types of source and 1 colour}

In this case we can distinguish say 2 types of source, with different evolutions, but we only measure total luminosities.

We have $J=2, j=1,2, I=1=i$, so all indices are source type indices. The constraints are

$\frac{L_{1}}{4 \pi \ell_{1}}=\frac{L_{2}}{4 \pi \ell_{2}}=\bar{d}_{\mathrm{L}}^{2}(z)$

This clearly constrains the relative evolution of the source types.

\subsection{Several types of source and several colours}

If we need to distinguish 2 (or more) source types, and we make multicolour observations, then we get many more constraints, and we improve our chances of demonstrating source evolution. If however the evolution of the source types is not very different, the extra effort would be unproductive. Indeed, the more types we distinguish and the more colours we use, the smaller the sample size in each redshift interval. However, with the large scale sky surveys now operating or being developed, this may not be much of a limitation. Nevertheless, identifying different source types requires resolved images or detailed spectroscopy for morphological classification, and involves considerable work.

\section{Conclusions}

- The main result is that measurements of apparent luminosities in various colours $\left\{\ell_{j i}(z)\right\}$ - ideally a set of spectral line intensities - put strong constraints on the colour evolution functions $\left\{L_{j i}(z)\right\}$. They determine all of the relative colour evolution functions $L_{j i}(z) / \bar{L}(z)$, but not $\bar{L}(z)$. The same applies to the apparent diameters. This allows source evolution theories to be tested against the observational data without any assumptions about homogeneity, or the cosmic equation of state, or knowledge of the true distances;

- The essential point is, although luminosity distances and diameter distances are model dependent, affected by both inhomogeneity and equation of state, their ratios in different colours are not;

- The converse is not true - multicolour observations do not directly help to pin down the degree of inhomogeneity. Nevertheless, once the absolute source evolution functions are reliably known from a well-confirmed galaxy evolution theory, using this and other methods, a fit of the observational data would be possible. Otherwise, we would still need to know the true distances, by an independent method, such as supernova light curves or gravitational microlensing, if they prove sufficiently reliable;

- Colour band number counts $c_{i}(z)$ by themselves (without luminosity measurements) do not put much constraint on candidate evolution theories, even if they are known versus redshift $z$. But, if they are summed over a range of $z$ values as is usual they tell us nothing;

- This method gives no direct information about the evolution quantities $m_{j}(z)$ and $\nu(z)$. However they are more amenable to determination through measurements of orbital velocities in galaxies, galaxy interactions, gravitational lensing surveys, etc. They will also be part of the evolution theories that are being tested by this method;

- The background model (LT) is not central to the ideas presented here. The idea of comparing observational relations in different colours or frequencies to obtain 
clear evidence of source evolution will obviously apply in any model;

- As already emphasised, it is important not to test source evolution theories by fitting to a homogeneous FLRW model, because then the possibility of detecting large scale inhomogeneity - or of demonstrating homogeneity - is removed, and the theories are wrong if there really is inhomogeneity;

- The approach suggested here offers the possibility of testing evolution theories independently of whatever inhomogeneity may be present, which is a distinct advantage over other methods. (However, the accurate determination of the inhomogeneity remains dependent on knowing the source evolution functions quite well.)

- It is worth emphasising that obtaining non-constant functions $L_{j i}(z) / \bar{L}(z)$ from multicolour observations does not itself prove the variation is due to time evolution - it could equally well be due to spatial variation. What it does do is provide data for testing theories of source evolution that is not contaminated by the gravitational effects of inhomogeneity in the intervening space;

- The ideal observations envisaged - a redshift value and at least two spectral line intensities on a large number of galaxies - may be possible, with large scale observing programs along the lines of the SDSS, or with the new generation of telescopes: Keck, Subaru, Gemini, VLT, SALT, etc. The $\mathrm{SDSS}^{15}$ is compiling an extensive and uniform database of galaxy data, for use in mapping the galaxy distribution, studying galaxy and quasar evolution and luminosity functions, improving the values of fundamental cosmological parameters, constraining dark matter distribution, and gravitational lensing studies. When completed, it will have photometrically recorded a quarter of the sky in 5 colour bands between 3000 \& 10000 angstroms. From this, 900000 galaxies with mean $z \approx 0.1$ plus 100000 luminous red galaxies with mean $z \approx 0.5$, and 100000 quasars, will have been selected for spectroscopic imaging;

- We advocate an observational program, possibly using SDSS data, if suitable, aimed at extracting the source evolution functions, and ultimately establishing the degree of large scale inhomogeneity, through the approach suggested here, as a very worthwhile complement to other methods being pursued. While there are plenty of practical problems involved - such as reddening, changing appearance of sources, etc. - that increase with $z$, they are all familiar or being faced in projects currently under study.

Acknowledgements. I am grateful to South Africa's NRF for a research grant. I thank George Ellis and the staff at the South African Astronomical Observatory, particularly Chris Koen, for helpful discussions, and I also thank Fernando de Felice for hospitality at the Dipartmento di Fisica of the Università degli Studi di Padova while this paper was completed.

\section{References}

Barrett, R. K., \& Clarkson, C. A. 2000, Class. Q. Grav., 17, 5047

Bell, E. F., \& Bower, R. G. 2000, MNRAS, 319, 235

Blundell, K. M., \& Rawlings, S. 1999, Nature, 399, 330

Bondi, H. 1947, MNRAS, 107, 410

Brunner, R. J., Szalay, A. S., \& Connolly, A. J. 2000, ApJ, 541, 527

Bullock, J. S., Dekel, A., Kolatt, T. S. et al. 2001, ApJ, 550, 21

Bunker, A., Spinrad, H., Stern, D. et al. 2000, in Proceedings of the Galaxies in the Young Universe II, Workshop, Ringberg Castle 2-6 August 1999, Springer-Verlag's Lecture Notes in Physics, ed. H. Hippelein, \& K. Meisenheimer

Cavaliere, A., \& Vittorini, V. 2000, ApJ, 543, 599

Celerier, M. N. 2000, A\&A, 353, 63

Dickinson, M. 2000, in Proceedings of the XIXth Moriond Astrophysics Meeting, Building Galaxies: From the Primordial Universe to the Present, March 1999, ed. F. Hammer, T. X. Thuan, V. Cayatte, et al. (Paris: Ed. Frontières), 257

Ellis, G. F. R., Perry, J. J., \& Sievers, A. W. 1984, AJ, 89, 1124

Ellis, R. S. 1997, ARA\&A, 35, 389

Ellis, R. S., Abraham, R. G., Brinchmann, J., \& Menanteau, F. 2000, Astron. Geophys., 41, 2.10-2.16

Fasano, G., Poggianti, B., Couch, W., et al. 2000, ApJ, 542, 673

Genzel, R., Lutz, D., \& Tacconi, L. 1998, Nature, 395, 859

Goodman, J. 1995, Phys. Rev. D, 52, 1821

Impey, C., \& Bothun, G. 1997, ARA\&A, 35, 267

Kennicutt, Jr., R. C. 1998, ARA\&A, 36, 189

Krasinski, A. 1997, Inhomogeneous Cosmological Models (Cambridge UP, Cambridge, ISBN 052148180 5)

Kodama, T., \& Bower, R. G. 2001, MNRAS, 321, 18

Lemaître, G. 1933, Ann. Soc. Scient. Bruxelles, A53, 51

Maor, I., Brustein, R., \& Steinhardt, P. J. 2000 [astro-ph/0007297]

Mustapha, N., Bassett, B. A. C. C., Hellaby, C., \& Ellis, G. F. R. 1998, Class. Q. Grav., 15, 2363 (MBHE)

Mustapha, N., Hellaby, C., \& Ellis, G. F. R. 1997, MNRAS, 292, 817 (MHE)

Perlmutter, S., Aldering, G., Goldhaber, G., et al. 1999, ApJ, 517,565

Ponman, T. J., Cannon, D. B., \& Navarro, J. F. 1999, Nature, 397,135

Riess, A. G., Filippenko, A. V., Challis, P., et al. 1998, AJ, 116, 1009

Riess, A. G., Filippenko, A. V., Li, W.-D., \& Schmidt, B. P. 1999, AJ, 118, 2668

Teerikorpi, P. 1997, ARA\&A, 35, 101

Tolman, R. C. 1934, Proc. Nat. Acad. Sci., 20, 169

Totani, T., \& Yoshii, Y. 2000, ApJ, 540, 81

Wang, Y., Spergel, D. N., \& Turner, E. L. 1998, ApJ, 498, 1

Watts, P., \& Taylor, A. 2000, MNRAS, 320, 139 